\title{
La Nuit des ondes gravitationnelles, une célébration scientifique et festive pour le grand public
}

Le CNRS et la Société Française de Physique ont organisé le 20 mars 2017 « La Nuit des ondes gravitationnelles » dans une quinzaine de villes de France et d'Italie. Avec des conférences scientifiques, des visites virtuelles de laboratoires, un quizz et une projection d'extraits de films commentés, ainsi que l'annonce des prix des différents concours lancés auprès des élèves et du grand public quelques mois plus tôt, cet événement ouvert à tous a célébré, de façon festive et avec une grande adhésion du nombreux public présent, la découverte des ondes gravitationnelles annoncée en février 2016 [1, 2].

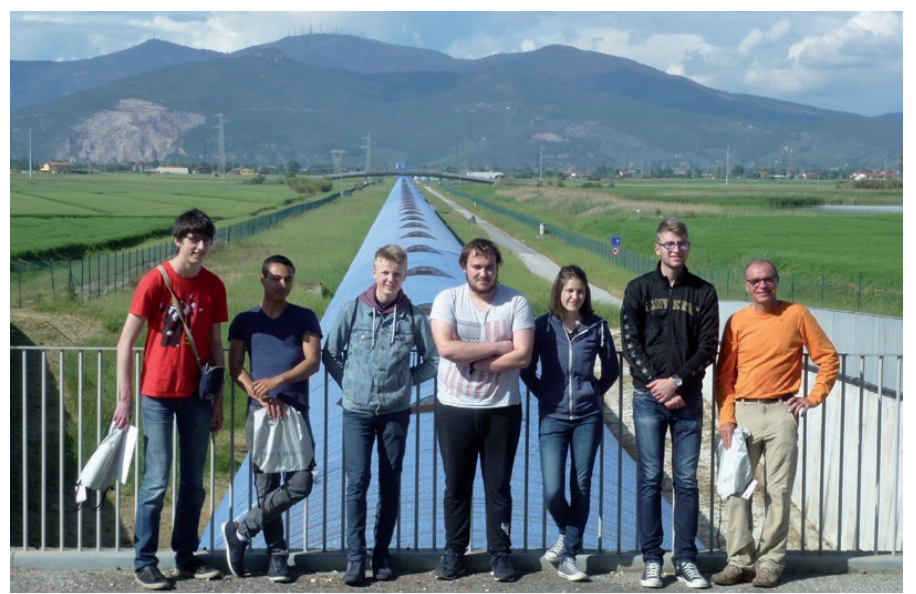

Les lauréats du concours « Entendre une onde gravitationnelle » (élèves de terminale $S$ du lycée Louis Aragon à Héricourt, Haute-Saône) visitant l'interféromètre Virgo.

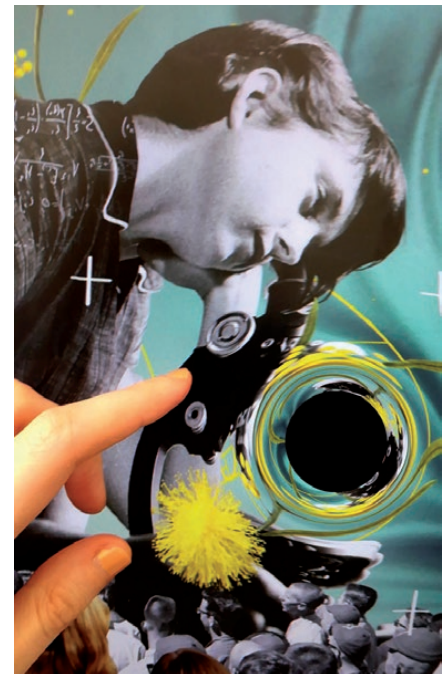

1. La photo « Comment détecter des phénomènes cataclysmiques via des signaux délicats ; comment voir au-delà des limites de la physique? " d'Audrey Hess, premier prix du concours "Photo dans l'œil du trou noir », catégorie « Tout public ».
La Nuit des ondes gravitationnelles a été l'occasion pour des lycéens, des étudiants, des enseignants et, plus généralement, pour de nombreuses personnes férues de science, de s'informer et d'échanger avec des scientifiques sur cette découverte majeure. Cette grande soirée festive s'est tenue simultanément dans onze villes en France et deux en Italie : Paris, Annecy, Bordeaux, Dunkerque, Limoges, Lyon, Lille, Nancy, Nice, Orléans, Strasbourg, Cascina (le site du détecteur Virgo, près de Pise) et Florence (à l'Institut Culturel Français).

En amont de cette soirée exceptionnelle, quatre concours mêlant art et science ont été organisés à destination des scolaires, des étudiants et du grand public :

- photos « déformées par un trou noir " (à l'aide de l'application pour téléphone portable "Pocket Black Hole", téléchargeable gratuitement [3]) (fig. 1) ;

- nouvelles ou bandes dessinées autour du thème des ondes gravitationnelles et des trous noirs (fig. 2);

- détection du signal caractéristique d'ondes gravitationnelles émis par la fusion de deux trous noirs, converti au préalable en son et dissimulé dans un environnement sonore bruyant ;

- génération "d'ondes gravitationnelles » : il s'agissait d'utiliser au mieux les analogies acoustiques, mécaniques, électromagnétiques, optiques des ondes gravitationnelles pour réaliser un montage original comportant quelques mesures.

Les concours techniques « détection et génération d'ondes gravitationnelles » n'ont pas rencontré d'écho notable dans le public, puisqu'à peine une petite dizaine de dossiers (au demeurant tous d'excellente qualité) ont été reçus. Par contre, les concours artistiques ont très bien fonctionné puisqu'au total une centaine de dossiers ont été déposés dans les différentes catégories. Le palmarès peut être consulté sur le site www.cnrs.fr/nuit-desondes-gravitationnelles, où l'on trouvera également le détail des présentations scientifiques données dans les différentes villes, les questions du quizz de milieu de soirée (décrit ci-dessous), ainsi que l'enregistrement vidéo de la conférence sur « La relativité au cinéma » à Paris.

La soirée du 20 mars a commencé à 18 h 30 par des exposés scientifiques dans des salles toutes pleines, rassemblant au total 5000 personnes, et dans une excellente ambiance. À Paris, au Grand Rex plein à ras bord avec ses 2700 places (!), la conférence a été animée par Alain Cirou, directeur de la rédaction du magazine Ciel et Espace, partenaire de l'événement. Les orateurs sont revenus sur la découverte des ondes gravitationnelles et sur les deux avancées majeures dont elle s'accompagne :

- d'une part la possibilité de détecter, grâce à des prouesses technologiques particulièrement ardues, des signaux très ténus ; 


\section{LES OAOES}

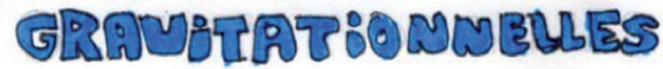
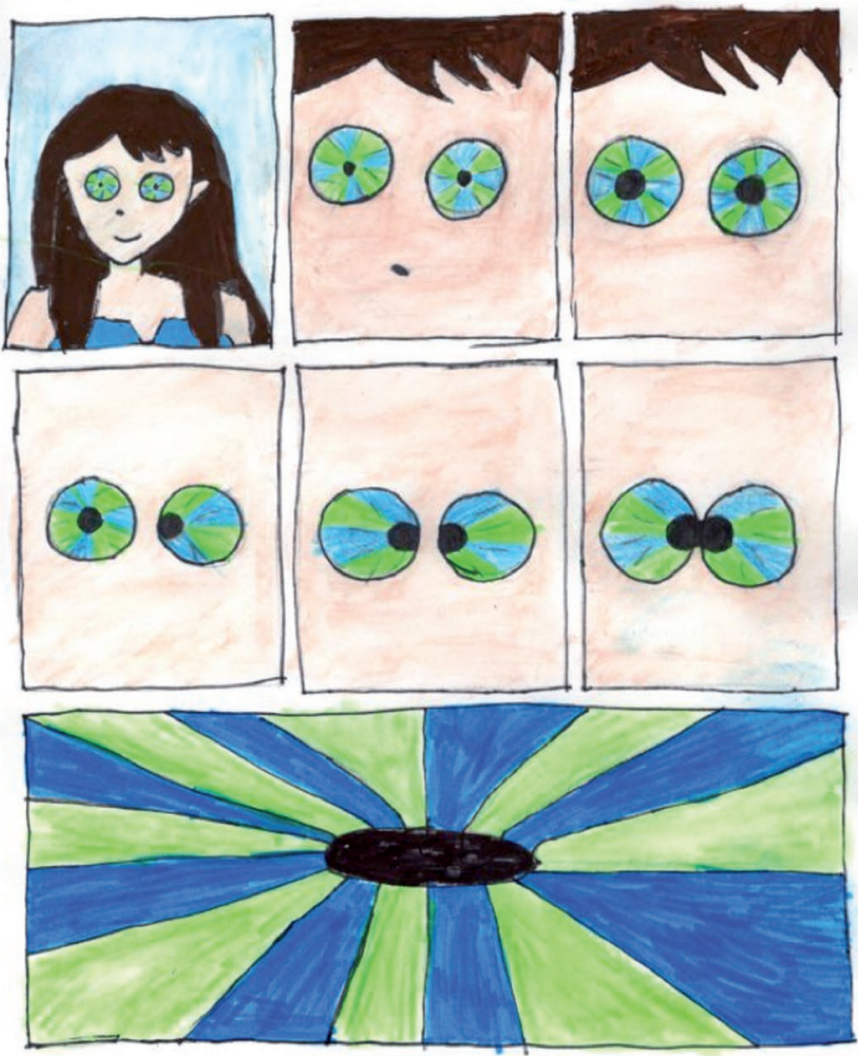

2. La bande dessinée « Les ondes gravitationnelles », réalisée par Coline Marsal (CM1) sous la houlette d'Audrey Dato (atelier de dessin de Quaix-en-Chartreuse, Isère), premier prix du concours " Nouvelles et bandes dessinées ».

c'est le résultat de projets au long cours, menés par des collaborations internationales grâce au soutien d'organismes de recherche - notamment le CNRS en France, l'INFN en Italie et la NSF aux États-Unis ;

- d'autre part, l'ouverture d'une nouvelle astronomie basée sur l'étude de phénomènes astrophysiques d'une violence inouie qui passaient auparavant complètement inaperçus ; et la possibilité de mener des tests encore plus poussés de la théorie de la relativité générale. À ce propos, il est remarquable que la découverte des ondes gravitationnelles ait eu lieu exactement 100 ans après leur prédiction par Albert Einstein !

Puis, vers 20 h 15, tous les sites ont été interconnectés par vidéo. Animée en direct depuis le Grand Rex par la "youtubeuse " Florence Porcel, cette deuxième partie de soirée aurait dû débuter par la visite en direct de la salle de contrôle du détecteur Virgo à Cascina (Italie). Cette expérience européenne (France, Italie, Pays-Bas, Pologne, Hongrie et Espagne), à laquelle participe le CNRS, est pleinement impliquée dans la découverte des ondes gravitationnelles au côté de son équivalent LIGO - dont les deux détecteurs sont aux États-Unis. Un problème technique n'a malheureusement pas permis de réaliser cette connexion, mais les spectateurs ont pu découvrir le cœur de l'interféromètre installé à Cascina, ainsi que les prouesses technologiques nécessaires à sa construction.

Le quizz était composé de sept questions comportant chacune quatre réponses possibles repérées par une couleur (jaune, rouge, verte ou bleue), certaines loufoques, d'autres beaucoup plus sérieuses. Chaque salle a pu voter au moyen de cartons colorés, disposés au préalable dans les salles - à raison d'un jeu de cartons pour deux spectateurs. Ce mécanisme a très bien fonctionné ; l'ambiance était partout électrique puisque des lots récompensaient les salles avec le meilleur total de bonnes réponses. Cinq villes sont arrivées en tête et ont été départagées par leur taille - la palme revenant à la plus petite d'entre elles. Dunkerque, Orléans et Strasbourg sont ainsi montées sur le podium.

Ensuite, un film a illustré les traitements de surface effectués au Laboratoire des Matériaux Avancés (LMA) de Lyon-Villeurbanne sur les miroirs de LIGO et de Virgo. Cette étape, d'une extrême technicité et qui demande une grande précision, est essentielle car les performances des détecteurs interférométriques d'ondes gravitationnelles (Virgo et LIGO) dépendent en grande partie de la qualité de leurs miroirs. Enfin, la soirée s'est conclue par une séquence "Science et cinéma " présentée par Richard Taillet (LAPTh-Université Savoie Mont Blanc) et Quentin Lazzaroto de l'Institut Henri Poincaré (IHP). Ils ont montré d'une façon à la fois amusante et profonde comment la relativité restreinte et la relativité générale ont été utilisées au cinéma dans de nombreux films de science-fiction de 1940 à nos jours, selon des angles d'approche divers qui ont été analysés avec finesse.

En conclusion, cette soirée a été très réussie, si l'on en juge par tous les commentaires reçus, à la fois dans les différentes salles mais aussi sur les réseaux sociaux, où notre "Nuit » a disputé la première place à un débat sur l'élection présidentielle programmé ce jour-là, et sur Internet où la soirée a été suivie en webcast par plusieurs milliers de personnes. Le public est manifestement très friand de ce type de manifestation et il appartient à notre communauté scientifique d'exploiter nos découvertes pour organiser d'autres « Nuits » du même genre qui brilleront d'autant de feux !

Nous tenons à remercier tous ceux qui se sont investis dans cette aventure : le CNRS, la Société Française de Physique, l'expérience Virgo et l'European Gravitational Observatory, et les laboratoires participants. Merci aux correspondants locaux de la SFP, sans qui il n'aurait pas été possible de faire participer autant de sites. I

Guy Wormser $^{(a)}$ et Nicolas Arnaud ${ }^{(b)}$

(a) Coordinateur de l'événement, Laboratoire de l'Accélérateur Linéaire (Orsay) (b) Laboratoire de l'Accélérateur Linéaire (Orsay) et European Gravitational Laboratory (Cascina)

\section{Références}

1 - L. Blanchet, "Les ondes gravitationnelles, cent ans après Einstein ", Reflets de la physique n52 (février 2017) 6-12.

2 N. Arnaud, "Les premières détections des ondes gravitationnelles", Reflets de la physique n52 (février 2017), 14-20.

3• www.laserlabs.org/pocketblackhole.php 